\title{
Requena Meana, Pablo. ¡Doctor, no HAGA TODO LO POSIBLE! DE LA LIMITACIÓN A LA PRUDENCIA TERAPÉUTICA
}

\section{EDITORIAL COMARES, S.L., COLECCIÓN BIBLIOTECA DE DERECHO Y CIENCIAS DE LA VIDA, GRANADA 2017 (ESPAÑA), 174 PÁGINAS, PRECIO 17 (RÚSTICA SIN SOLAPAS), ISBN13: 978-84-9045-573-9}

Fermín J. González-Melado*

DOI: $10.5294 /$ pebi.2018.22.1.12

\section{¡DOCTOR, NO HAGA TODO LO POSIBLE!}

Pablo Requena ha titulado su libro con una sugestiva frase ¡Doctor, no haga todo lo posible! El título recoge una realidad presente hoy en muchos de nuestros hospitales. Los profesionales de la medicina han sido educados para luchar contra la enfermedad con todas sus fuerzas y recursos. Sin embargo, en últimos decenios, la medicina ha vivido una revolución en sus posibilidades diagnósticas y terapéuticas que ha hecho que en muchas ocasiones ese hacer "todo lo posible" no sea lo mejor que el personal sanitario pueda ofrecer a su paciente.
Aunque se ha reflexionado mucho desde el punto de vista teórico sobre la necesidad de limitar determinados tratamientos, lo cierto es que en la práctica cotidiana hospitalaria la limitación de un tratamiento sigue siendo percibida por muchos como un cierto abandono del paciente. Éste es el contexto en el que se sitúa el autor y ese contexto marca el objetivo de este libro: ayudar a aquellas personas que, cada día, deben tomar decisiones difíciles, relacionadas con la vida y la muerte de sus pacientes. El libro, por tanto, tiene la pretensión de ser un instrumento práctico: el autor propone un modo de proceder que ayude a los agentes morales implicados,

* $\quad$ orcid.org/0000-0001-8975-069X. Centro Superior de Estudios Teológicos, Badajoz, España. ferminjgm@hotmail.com 
médico-paciente (familia), a tomar de manera compartida, cuando sea posible, la decisión correcta en cada caso concreto.

Por eso los principales destinatarios de este libro son los profesionales de la salud que se enfrentas a situaciones clínicas en las que las opciones terapéuticas son variadas y se precisan decisiones técnica y moralmente válidas. También va dirigido a los juristas que tanto desde el punto de vista teórico (legislación) como desde el punto de vista práctico (resolución de conflictos) tienen que afrontar y resolver situaciones de LET. Y, por último, otros destinatarios son los especialistas de ética médica y bioética que abordan estas cuestiones en los debates académicos de estas disciplinas.

Tras el prólogo inicial nos encontramos con la introducción del autor, seis capítulos y un último apartado donde se recogen algunas conclusiones. Aunque el autor no lo hace, podemos dividir el libro en dos partes.

\section{PRIMERA PARTE DEL LIBRO: LA CIENCIA MÉDICA DE LA LET}

Esta primera parte abarca los tres primeros capítulos en los que el autor hace un análisis estrictamente médico de lo que significa la limitación del esfuerzo terapéutico (LET).

En el primer capítulo, enmarca los límites de la buena praxis médica, dónde se tiene que mover la LET. El límite inferior vendría marcado por el daño por defecto, es decir el abandono terapéutico del paciente. El autor analiza en profundidad las situaciones extremas de este límite inferior que son la eutanasia y el suicidio asistido. El límite superior, o daño por exceso de atención, vendría delimitado por las terapias fútiles y la llamada obstinación terapéutica. Lo curioso, como señala el autor, es que estos dos extremos tienen una misma raíz, la idea de eficiencia a toda costa: "los grandes avances tanto en el diagnóstico como en las terapias han hecho que se olvide que la muerte del paciente no es siempre un mal resultado del ejercicio clínico. Lo que sería un mal resultado sería no evitar, cuando sea posible, una 'mala muerte""(1). En este primer capítulo destaca el apartado 3.- La "interrupción voluntaria del comer y beber". El autor estudia una realidad que no es muy frecuente en el ámbito de habla española y que sí es frecuente en el ámbito anglosajón: la "voluntary stopping of eating and drinking” (VSED). La literatura anglosajona recoge que la VSED sería una de las últimas opciones a ofrecer por los cuidados paliativos en los momentos finales. Como tanto el suicidio asistido y la eutanasia voluntaria están legalmente prohibidas en muchos países, la VSED, junto con la sedación terminal, aparece como una opción válida para pacientes terminales que quieren poner fin a su vida. Me parece muy interesante este apartado porque, aunque no está muy presente en el ámbito latinoamericano, si es cierto que la influencia anglosajona hará que pronto, en muchos de nuestros hospitales, se presente la VSED como una posibilidad al final de la vida, y tenemos que ser conscientes de que la elección por parte del paciente de interrumpir la alimentación y la bebida de manera voluntaria no es otra cosa que una forma de suicidio.

El segundo capítulo del libro hace una presentación general del fenómeno de la LET. Partiendo de la historia reciente sobre limitación de algunos tratamientos como las órdenes de no resucitación o la suspensión de la ventilación mecánica, el autor hace un planteamiento muy original al hacer un estudio epidemiológico de la LET, 
es decir, analiza la frecuencia de toma de decisiones de LET en el ámbito hospitalario. A partir de ahí se centra en las definiciones de LET para aislar tres elementos presentes en toda definición de LET: a) el tipo de acción que se realiza (detener o no iniciar); b) lo que se limita (la terapia) y c) el motivo por el que se hace (futilidad). Dentro de este capítulo, llama la atención cómo dentro de un contexto hospitalario marcado por la importancia de la autonomía del paciente los datos revelan que son los médicos los responsables finales de las decisiones LET. $\mathrm{El}$ autor aboga por la vía de la "decisión compartida". Está claro que el problema más difícil se encuentra en los casos de pacientes no competentes o que no quieren afrontar el tema. El autor plantea un proceso de toma de decisiones en tres fases: a) un primer momento que corresponde al médico y que sería un juicio técnico sobre la oportunidad clínica de ese tratamiento; b) una segunda fase correspondería al juicio de lo adecuado de ese tratamiento a la situación concreta del paciente; a partir de aquí se puede llegar a la conclusión de que el tratamiento es fútil y por tanto no se debe iniciar o, en el caso de iniciado suspenderlo. Si el tratamiento se considera adecuado todavía quedaría c) un tercer paso en el que el propio paciente debe decidir si está dispuesto o no a llevar adelante el tratamiento.

En el tercer capítulo, el autor selecciona una serie de tratamientos en los se plantea de manera más frecuente la LET: la reanimación cardiorrespiratoria, el soporte ventilatorio, la hemodiálisis, la quimioterapia, la hidronutrición por vía artificial y el ingreso en la unidad de cuidados intensivos (UCI). Es el capítulo más extenso de todo el libro y es el que más se ve el esfuerzo del autor por conseguir que el libro sea un instrumento práctico en manos de los profesionales sanitarios. El autor plantea diversos escenarios y situaciones a las que se enfrentan normalmente el médico, y estudia los criterios que normalmente se utilizan en las LET en estas situaciones, como la enfermedad de base, el pronóstico vital, la edad del paciente, etc. De este tercer capítulo el apartado más interesante es el referido a la decisión de ingreso del paciente en la UCI. La negación de un paciente al ingreso en UCI es una LET particular no solo porque hace referencia a un conjunto de medidas que el paciente no va a recibir, sino porque algunos casos la negación del ingreso en UCI se debe a la no disponibilidad de camas, es decir a un problema de justicia. Esta peculiariedad, lleva al autor a distinguir entre criterios objetivos (referidos al juicio prudencial sobre el beneficio sustancial que recibiría el paciente si ingresa en UCI) y criterios circunstanciales (referidos a una cuestión de justicia a la hora de asignar los recursos disponibles).

Echamos en falta que en este tercer capítulo no se haga una presentación de la toma de decisiones LET en el ámbito de las unidades de neonatología, las llamadas UCIs pediátricas. Éste es uno de los contextos actuales en los que se ha recrudecido el debate bioético y donde aparecen una serie de conceptos como el de "ventana de oportunidades" (2-3), a la hora de no iniciar tratamientos de soporte vital, que están siendo muy controvertidos. Además, el ámbito pediátrico pone de manifiesto como muchas veces no es posible llegar a acuerdos entre los médicos y los padres ${ }^{1}$, y las decisiones de LET se con-

1 Un caso reciente es el del bebé Charile Gard, cuyos padres recurrieron la decisión de los médicos de suspender los tratamientos de soporte respiratorios. El caso tuvo una amplia repercusión mediática. [https://elpais.com/internacional/2017/07/28/actualidad/1501263240_986226.html] (acceso 13 febrero 2018) 
vierten en una fuente de conflictos sobre lo que significa el mejor interés del niño (4).

\section{3.- SEGUNDA PARTE DEL LIBRO: LA CIENCIA ÉTICA DE LA LET}

Los tres siguientes capítulos, que conformarían esa hipotética segunda parte, recogen la reflexión ética y la aportación específica del autor al tema en cuestión. Bajo el título "La "aceleración" de la muerte: entre intenciones y causalidad", el capítulo cuarto denuncia la poca reflexión a la hora de identificar los distintos tipos de acciones que se llevan en los momentos del final de la vida. Es el capítulo de intersección entre el nivel general, que hemos observado en los tres primeros capítulos, y los juicios particulares de conciencia ante la situación del paciente concreto. Por eso este capítulo está centrado en el estudio de aquellas categorías que pueden ayudar a los agentes morales (médicos, paciente y familia) en la identificación de los distintos tipos de acción al final de la vida. El autor comienza clarificando un concepto, el de "aceleración" de la muerte, que siendo ampliamente en la actualidad, sin embargo, no tiene un sentido unívoco. Partiendo de que toda actuación médica modifica los tiempos del morir, el autor se centra en la intencionalidad de la acción médica para hacer la valoración moral de cada decisión. Por eso recomienda no usar la expresión "acelerar la muerte" como categoría moral o, al menos, distinguir dos sentidos: uno lato sensu, en sentido general y sin pretensión moral, y otro en stricto sensus, como sinónimo de eutanasia, es decir, que la acción se elige para terminar con la vida del paciente. Posteriormente, el autor hace un amplio análisis de los dos niveles de intencionalidad en las decisiones al final de la vida. En realidad, este segundo apartado del cuarto capítulo es todo un tratado de teoría de la acción. A partir del ejemplo de la sedación terminal, el autor determina dos niveles de intencionalidad: uno relativo al fin del agente y otro que se refiere a los medios que se eligen para llegar a ese fin. La elección de los medios para conseguir el fin es lo que en la tradición moral se ha denominado "objeto moral" de la acción, lo que Rhonheimer denomina "acción intencional básica” (5) y es el que permite la primera valoración moral de la acción. En el tercer apartado el autor entra en el problema de la causalidad de la acción y su consecuencia, y la responsabilidad moral del agente por dicha acción. Comienza clarificando la distinción entre efectos queridos y efectos previstos de una acción. Frente a la vía de sopesar las consecuencias de una acción para valorar su moralidad, propia del utilitarismo, el autor opta por estudiar el proceso intencional del obrar para valorar la acción, más propio de una ética de la virtud. La no distinción que hace el utilitarismo entre efectos deseados y efectos previstos lleva a los utilitaristas al proporcionalismo moral, donde siempre existirá un motivo proporcionado para justificar cualquier decisión. A la hora de valorar moralmente una acción de omisión el autor se sitúa entre los extremos de las éticas utilitaristas (6) y de las éticas deontológicas (7), para proponer la ética de la virtud por la que el médico de manera natural, y no por obediencia a una norma externa, pondrá en marcha un proceso de discernimiento a través de su racionalidad práctica, no solo sobre la futilidad de un tratamiento, sino también sobre si el medio terapéutico supone una carga muy pesada para el paciente o para los que se encargan de su cuidado. El último apartado de este cuarto capítulo viene a recordarnos la equivalencia moral que existe entre "no iniciar" y "suspender" una medida terapéutica. Hoy en día en el ámbito bioético existe una aceptación prácticamente unánime de la tesis de equivalencia moral entre "no iniciar" y "suspender" un tratamiento, sin embargo, no ocurre lo mismo entre 
los profesionales sanitarios que siguen estableciendo una diferencia moral entre ambas acciones. Por eso el autor recuerda que la valoración moral de las decisiones que se realizan al final de la vida no depende de que la acción elegida sea "no iniciar" o "suspender" sino que es necesario identificar los niveles de intencionalidad y estudiar las conexiones causales de las acciones realizadas y sus consecuencias.

En el quinto capítulo, titulado "Categorías para la valoración moral”, el autor se centra en el análisis de aquellas categorías que la bibliografía médica y bioética utilizan para la justificación moral de la LET: la autonomía del paciente, la calidad de vida y la proporcionalidad terapéutica. Lo que trata el autor es saber si estas categorías son válidas a la hora de ofrecer una justificación adecuada de una decisión de LET. En el caso de la autonomía, frente a la dialéctica autonomía del paciente - paternalismo médico, el autor propone un proceso de "decisión compartida". El argumento de la calidad de vida es el más utilizado para justificar las decisiones de LET, sin embargo, el autor demuestra como la utilización, aparentemente objetiva, del criterio de calidad de vida va contra la propia autonomía del paciente, porque en situaciones médicas similares, enfermos con distintos valores y perspectivas de la vida elegirían acciones distintas. Por eso el autor prefiere que las decisiones LET se justifiquen desde un desde un cálculo de riesgos, costes (también los físicos y psíquicos) y los posibles beneficios, después de haber tenido en cuenta las preferencias del propio paciente. En el tercer apartado de este quinto capítulo está dedicado a un amplio estudio sobre el concepto de proporcionalidad terapéutica. El autor, partiendo de la distinción clásica de medios ordinarios y extraordinarios, hace un análisis de la distinción actual entre medios proporcionados y desproporcionados y, más concretamente, de la propuesta de Calipari (8) de aplicación a la práctica clínica. El autor concluye que, si bien la distinción clásica entre medios ordinarios y extraordinarios, podría ser válida para justificar la LET, en el contexto actual podría ser insuficiente y que sería más completo un análisis de los elementos que configuran la acción, como ya describió el autor en el cuarto capítulo. El último apartado de este capítulo el autor propone una combinación de perspectivas: la perspectiva del ethos médico (representada por el principio de proporcionalidad terapéutica) y la perspectiva de la vida del paciente (representada por el concepto de calidad de vida). Esta combinación solo es posible cuando superamos la dialéctica entre ambas a través de la "decisión médica compartida", que es una de las propuestas clave del autor en éste libro.

El sexto capítulo recoge la aportación de síntesis del autor ante el problema de la LET: la necesidad de la virtud de la prudencia por parte del médico y la importancia de la comunicación médico-paciente (familia) a la hora de tomar decisiones de LET. La prudencia vendría definida como la virtud que es capaz de llenar el espacio que se abre entre el conocimiento ético general y la actuación concreta. Frente a la corrupción que el término prudencia ha vivido en el ámbito médico, reduciéndola a una cierta habilidad técnica (hacer buenos diagnósticos y dar buenos tratamientos) el autor defiende la recuperación por parte del médico de la prudencia como virtud de la acción, que se concentra en la elección de las acciones teniendo en cuenta el bien global del paciente. Siguiendo a Pieper distingue las dos dimensiones principales de la virtud: la dimensión cognoscitiva y la dimensión imperativa (9). Esta distinción sirve al autor para plantear que la valoración moral sobre decisiones 
de LET no debe quedar reducida al análisis de la acción concreta, sino que incluye la valoración moral del proceso para llegar, en esa situación concreta, a la mejor decisión. La buena realización del trabajo médico exige siempre, pero más en estas decisiones de LET, no solo competencia técnica sino el auxilio de las virtudes de justicia, fortaleza y templanza. El segundo apartado de este capítulo aborda la importancia de la comunicación en la relación médico-paciente. En el contexto sanitario actual, donde se multiplican las figuras profesionales que atienden al paciente, éste tiene que tomar decisiones muy importantes sobre su vida y su muerte con personas (médicos) que le resultan, la mayoría de las veces, prácticamente desconocidos. Por eso el autor subraya en este apartado la necesidad que tienen los médicos de desarrollar ciertas habilidades de comunicación. Frente a lo que se ha dado en llamar "el silencioso mundo del médico y su paciente"2 (10) el autor propone un cambio de paradigma que pasa por la necesidad de una oportuna formación de los futuros profesionales de la medicina. El contacto de los estudiantes con los pacientes desde los primeros cursos para desarrollar sus capacidades de comunicación, la valoración de las LET como decisiones positivas cuando no se ve razonable seguir insistiendo con un tratamiento o la importancia de los tiempos necesarios para que el paciente asimile la información, son elementos indispensables de ese cambio de paradigma que lleve a la asunción de decisiones compartidas por el médico y el paciente (o sus familiares) en todos los tratamientos y también en las decisiones de final de vida.

2 Con ésta expresión, Katz, en "The Silent World of Doctor and Patient”, recoge la conciencia que tienen los médicos de no involucrar demasiado al paciente, o a los familiares, en las decisiones de final de vida, para evitarles cargar con el peso de la decisión.
Al final del libro aparece un apartado de Algunas conclusiones donde el autor recoge algunas de las aportaciones de su trabajo. El análisis epidemiológico de la LET demuestra que es una acción clínica frecuente en el ámbito hospitalario, especialmente en las UCI, y por lo tanto que es una cuestión ética importante. Lo que no resulta siempre fácil es justificar éticamente esas decisiones. Muchas LET se apoyan sobre los conceptos de autonomía y de calidad de vida, que el autor ha demostrado que, por sí mismos, no son válidos para justificar ese tipo de decisiones. El autor considera que sigue siendo válida la distinción entre medios ordinarios y extraordinarios y el principio de proporcionalidad terapéutica, dentro de un proceso de decisión compartida entre médicopaciente. La virtud de la prudencia terapéutica facilitará al médico y al paciente esa decisión que será posible si se cuida la comunicación entre ambos. Por ello, una de las conclusiones finales del trabajo es la necesidad de enseñar y promover las habilidades de la comunicación a los profesionales sanitarios.

\section{4.- VALORACIÓN CRÍTICA DEL LIBRO}

Las decisiones médicas al final de la vida son unos de los temas más actuales en la bioética. La defensa a una muerte digna ha llevado a la consideración de que la eutanasia y el suicidio médicamente asistido son las únicas vías de solución para un paciente terminal. Los cuidados paliativos aparecen con fuerza como una alternativa eficaz en estas situaciones, pero también la LET es una opción positiva cuando las cargas de un determinado tratamiento son insoportables para el paciente o sus familiares. De eso es precisamente de lo que trata este libro, de presentar las decisiones de LET como decisiones que forman parte de la prudencia terapéutica de la medicina, es decir, del obrar del "buen médico". 
Desde ese planteamiento el libro tiene un objetivo eminentemente práctico que lo hace muy valioso: ayudar a los profesionales que tienen que tomar este tipo de decisiones no solo para que elijan siempre la decisión éticamente correcta, que en muchas ocasiones será muy difícil, sino para que les quede la tranquilidad de que la manera en la que llegaron a tomar esa decisión sí que fue la más adecuada. El autor distingue a la hora de decidir una LET entre un primer momento de tipo médico donde entraría no solo la evaluación de futilidad del tratamiento sino su adecuación para la situación concreta de este paciente concreto, considerando el bien global del paciente. Es esta primera fase la que requiere la prudencia terapéutica del médico. Un segundo momento de la decisión correspondería a la evaluación que el propio paciente y su familia hacen de las cargas físicas y psíquicas que el tratamiento tiene. Tanto en el primer momento como en el segundo momento la comunicación médico-paciente deben ser la base de una “decisión compartida” acerca de la idoneidad de la LET.

Este deseo de ser instrumento práctico se ve también en la clarificación de las situaciones clínicas en las que se plantean las decisiones de LET (capítulo tercero) así como la profundización en la teoría de la acción (capítulo cuarto). El autor quiere, de verdad, ayudar a comprender a cada médico los elementos intencionales y las relaciones causales que se encuentran en sus decisiones de LET y así poder evaluarlas éticamente. No es en vano que los capítulos tercero y cuarto sean los más amplios del libro.

La abundante bibliografía tanto en lengua inglesa como en lengua española permiten al autor un análisis exhaustivo tanto a nivel técnico-medico como a nivel de reflexión ética. Es también de agradecer la soltura con la que el autor se mueve tanto en la parte científico-médica como en la parte ético-moral. El dominio de estos dos ámbitos (el autor es licenciado en medicina, doctor en teología y especialista en teología moral y bioética) hace que la lectura del libro tenga una coherencia estructural y que, a pesar de la división de capítulos, mantenga una estructura lógica y un lenguaje asequible tanto para médicos, como para juristas y para especialistas en moral. Hay que hacer una llamada al autor a la utilización, en algunos momentos, de una división de los apartados en subapartados que ayuden al lector a centrar la reflexión, especialmente en aquellos apartados más amplios como, por ejemplo, el apartado dos del capítulo quinto sobre los niveles de intencionalidad.

Más allá del estudio concreto de la valoración moral de las decisiones de la LET, Pablo Requena aboga en este libro por un cambio de paradigma en las relaciones médico-paciente. Huyendo de la dialéctica autonomía del paciente - paternalismo médico, Requena defiende un nuevo sistema de relaciones basado en la decisión compartida. Para ello el autor ve necesarios dos elementos fundamentales. El primero es la configuración del médico no solo desde la pericia técnica sino también desde la excelencia ética. En este sentido la importancia de la prudencia terapéutica como virtud médica configura todo el ser del médico. El médico virtuoso no es solo necesario para afrontar las decisiones de LET sino que la virtud de la prudencia configura al buen médico en todas sus acciones cotidianas: en la relación con los pacientes, con la familia del paciente y con otros profesionales sanitarios. El segundo elemento es la importancia de la comunicación con el paciente y con sus familiares a la hora de tomar decisiones. Está claro que en la formación de los estudiantes de medicina hay un déficit en lo que se refiere al aprendizaje de estrategias 
de comunicación y a la percepción de que la manera de comunicarse con el paciente es parte importante de su cualificación médica. Es necesario que los estudiantes de medicina sean conscientes de estos dos elementos, la prudencia terapéutica y la importancia de la comunicación, en su proceso de formación no solo para ser buenos profesionales sino para llegar a ser "buenos médicos". Por ellos creemos que, junto con los destinatarios ya comentados por el autor, médicos, juristas y expertos de bioética, los estudiantes de medicina son unos destinatarios privilegiados a los que este libro les podrá ayudar en el camino a la excelencia médica que pasa, necesariamente, por la excelencia ética.

\section{REFERENCIAS}

1. Requena, P. jDoctor no haga todo lo posible! De la limitación a la prudencia terapéutica. Editorial Comares, Granada 2017.

2. Wilkinson D. The window of opportunity: decision theory and the timing of prognostic tests for newborn infants. Bioethics. 2009; 23: 503-14.
3. Wilkinson D. Death or Disability? The "Carmentis Machine" and decision-making for critically ill children. Oxford: Oxford University Press; 2013. 320 p.

4. González-Melado FJ, Di Pietro ML. El mejor interés del niño en neonatología: ¿qué es lo mejor para el niño?. Cuadernos de Bioética. 2015; 26: 201-22.

5. Rhonheimer M. La perspectiva de la moral: fundamentos de la ética filosófica. Madrid: Rialp; 2000. 456 p.

6. Kuhse H. Sanctity-of-life doctrine medicine: a critique. Oxford: Clarendon Press; 1987. 256 p.

7. Ferguson PR. Causing death or allowing to die? A rejoinder to Randall's comments. Journal of Medical Ethics. 1998; 24: 281-82.

8. Calipari M. Curarse y hacerse curar: entre el abandono del paciente y el encarnizamiento terapéutico. Ética del uso de los medios terapéuticos y de soporte vital. Buenos Aires: EDUCA; 2007. 232 p.

9. Pieper J. Prudencia y templanza. Madrid: Rialp; 1969. 228 p.

10. Katz J. The silent world of doctor and patient. Baltimore: Johns Hpkins University Press; 2002. 320 p. 